\title{
The opioid epidemic and intravenous drug-associated endocarditis: A path forward
}

Chris C. Cook, MD, ${ }^{\mathrm{a}}$ J. Scott Rankin, MD, ${ }^{\mathrm{a}}$ Harold G. Roberts, MD, ${ }^{\mathrm{a}}$ Gorav Ailawadi, MD,

Mark Slaughter, MD, ${ }^{\mathrm{c}}$ Lawrence M. Wei, MD, ${ }^{\mathrm{a}}$ and Vinay Badhwar, MD ${ }^{\mathrm{a}}$

\footnotetext{
From the a Department of Cardiovascular and Thoracic Surgery, West Virginia University, Morgantown, WVa; ${ }^{\mathrm{b}}$ Division of Cardiac and Thoracic Surgery, University of Virginia, Charlottesville, Va; and ${ }^{\mathrm{c} D e p a r t m e n t ~ o f ~ C a r-~}$ diovascular and Thoracic Surgery, University of Louisville, Louisville, Ky.

Received for publication June 16, 2019; revisions received Aug 4, 2019; accepted for publication Aug 6, 2019; available ahead of print Oct 15, 2019.

Address for reprints: Vinay Badhwar, MD, Department of Cardiovascular and Thoracic Surgery, West Virginia University, 1 Medical Center Drive, Morgantown, WV 26506 (E-mail: vinay.badhwar@wvumedicine.org). J Thorac Cardiovasc Surg 2020;159:1273-8 $0022-5223 / \$ 36.00$

Copyright (C) 2019 Published by Elsevier Inc. on behalf of The American Association for Thoracic Surgery https://doi.org/10.1016/j.jtcvs.2019.08.112
}

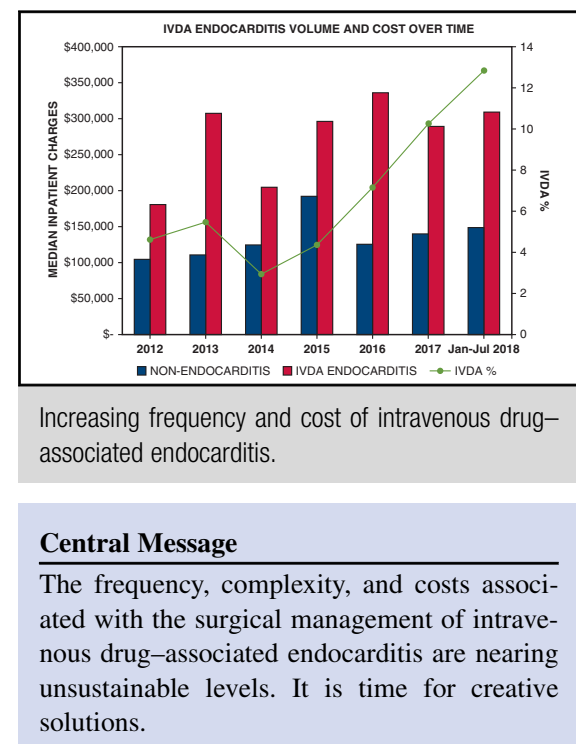

This Invited Expert Opinion provides a perspective on the following paper: $N$ Engl $J$ Med. 2019;380:415-24. https://doi.org/10. 1056/NEJMoa1808312.

\section{THE SCOPE OF THE PROBLEM}

The US opioid epidemic has been implicated in substantial rises in mortality, morbidity, and costs. According to the Centers for Disease Control and Prevention, between 1999 and 2017 there were nearly 218,000 opioid-related deaths in the US, 47,600 in 2017 alone. ${ }^{1}$ The staggering rise in recent years is illustrated by a $9.6 \%$ increase in the age-adjusted rate of overdose deaths just from 2016 to 2017. ${ }^{1}$ States with the highest rates of death due to drug overdose, the majority from intravenous (IV) heroin use, were West Virginia (57.8 per 100,000), Ohio (46.3 per 100,000), Pennsylvania (44.3 per 100,000), the District of Columbia (44.0 per 100,000), and Kentucky (37.2 per 100,000). ${ }^{1-3}$ The increasing rate of drug overdose death has been accompanied by a commensurate increase in the incidence of IV drug-associated (IVDA) acute infective endocarditis. ${ }^{4}$

Although the opioid epidemic has become a global phenomenon, the United States leads the world in opioid consumption, abuse, and opioid-related deaths. ${ }^{5}$ How did we get to this point? Several key elements have converged in recent years to produce this crisis. The epidemic's first wave began with the rampant overprescribing of opioid pain medication between 1995 and 2005, resulting in an overwhelming number of opioid-dependent patients. Aided by a long-cited belief that physicians commonly failed to provide adequate pain relief, 6 and reports of a low risk of addiction in the treatment of cancer patients, ${ }^{7}$ the accelerated use of prescription narcotics for the treatment of chronic noncancer pain began in the late 1980s to early 1990s. In 1995, 2 very important factors emerged: the introduction of OxyContin (Purdue Pharma, Stamford, Conn) and a related campaign dubbed "Pain is the Fifth Vital Sign". ", Following the launch of the Pain is the Fifth Vital Sign campaign at the annual American Pain Society meeting in 1995, the American Academy of Pain Medicine issued a consensus statement on the use of opioids in treating chronic pain suggesting it to be as equally important as traditional vital signs. ${ }^{8}$ In the ensuing years, more than 20,000 industry-funded educational programs were held to break down physicians' barriers to aggressive pain treatment. ${ }^{9}$

The added pressure to alleviate pain at all costs, coupled with the aggressive marketing campaigns of pharmaceutical manufacturers, produced a growing population of opioid addicts. The hardest-hit communities were those of low socioeconomic status, particularly in the Appalachian region of the United States. ${ }^{10,11}$ The overprescription of OxyContin in these communities provided abundant access for illicit recreational use. The resale of OxyContin, colloquially termed "hillbilly heroin," grew rapidly. The American Academy of Pain Medicine and the American Society of Addiction Medicine issued their first warnings on the risk of overprescription of opioids in $2004 .^{12}$

The second wave of the epidemic occurred in 2010. Patients who could no longer obtain prescription narcotics began to turn to an increasingly-affordable influx of illicit 
drugs, especially heroin. As many as 4 out of 5 heroin users report having started with nonmedical use of prescription opioid pain medications. ${ }^{13}$ The third wave of the epidemic hit in 2013 with the introduction and increased trafficking of illicitly manufactured fentanyl, which is $30-50$ times more powerful than heroin.

This crisis is not limited to the socioeconomically disenfranchised, as illustrated by the recent deaths of prominent musicians succumbing from fentanyl overdose (eg, Prince, Tom Petty, Mac Miller). Although legislative efforts aimed at mandated prescriber education and monitoring programs have attempted to curtail the excessive use of narcotics, the widespread availability of inexpensive heroin, the influx of illicit fentanyl, and the socioeconomic factors that continue to plague many communities prone to IV drug abuse have left the increased incidence and sequelae of this abuse, such as acute endocarditis, to go largely unabated. These factors have tremendous impacts on cardiac surgery and healthcare, especially in high-risk areas like the Appalachian region of the United States..$^{10,11,13,14}$

\section{EVOLVING TRENDS AND COSTS FROM THE FRONT LINES OF THE OPIOID CRISIS}

The clinical impact of the opioid crisis can be demonstrated by the exponential rise in the incidence and costs associated with IVDA endocarditis. To illustrate this issue, recent trends in patients with acute IVDA tricuspid valve (TV) endocarditis presenting to West Virginia University were examined. Patients with a diagnosis of IVDA endocarditis undergoing TV operations between January 2012 and December 2018 were examined (Table 1). From 2012 to 2018, the average annual increase in IVDA TV operations was $226 \%$ (Figure 1). The majority of these operations for IVDA TV endocarditis occurred between 2016 and 2018 (88\%), with 5 cases in 2012 and 84 cases in 2018. No patient underwent valvectomy. Isolated TV operations were performed in $72 \%$ of cases (108 of 149), and $11 \%$ were performed via minimally invasive thoracotomy using peripheral cannulation. Before 2016, the primary operative technique was TV replacement. From 2016 to 2018, repair and replacement techniques were applied equally (65 TV repair [49.6\%] vs 66 TV replacement [50.4\%]; $P=.930$ ). Mortality at 30 days was $0 \%$ in $2016,3 \%$ in 2017, and 4\% in 2018 (Figure 1). The median hospital length of stay (LOS) increased from 7 days in 2012 to 35 days in $2018(P=.008)$ with implementation of an institutional policy of inpatient completion of IV antibiotic administration to combat outpatient indwelling catheterrelated acute recidivism. Surgical adaptation appears to be necessary to combat an exponential rise in the incidence and complexity associated with acute IVDA TV endocarditis. However, acceptable outcomes appear to be possible with the application of TV repair and patch reconstruction, as well as minimally invasive techniques for this once rare but now all too common problem.

Costs associated with IVDA operations performed at West Virginia University were also examined. Patients undergoing valve operations with IVDA endocarditis $(n=187)$ and without IVDA endocarditis $(n=613)$ between January 1, 2012, and July 1, 2018, were examined. Total actual inpatient charges, reimbursements, and postoperative LOS were examined. Patients with IVDA endocarditis composed $5 \%$ of the total cardiac operative volume in 2012 and $13 \%$ in 2018 (Figure 2), which included $10 \%$ of all cardiac cases from July 2016 to July 2018. Compared with valve operations without endocarditis, the patients with IVDA-related endocarditis were younger (median age, 34 years vs 64 years; $P<.001$ ), had a greater prevalence of tricuspid valve surgery $(55 \%$ vs $9 \% ; P<.001)$, a greater need for postoperative noncardiac operations $(9 \%$ vs $3 \% ; P<.001)$, and higher composite major morbidity/mortality $(31 \%$ vs $16 \% ; P<.001)$ (Table 2). Furthermore, noncardiac operations were required more frequently in the IVDA-related group, including a greater need for dental extractions as well as interventions to treat embolic infections: amputation, drainage of joint or spinal abscesses, splenectomy, brain abscess, cholecystectomy, and thoracoscopy for empyema.

TABLE 1. Trends and outcomes of surgical treatment of IVDA TV endocarditis at West Virginia University

\begin{tabular}{lcccccc}
\hline Year & Cases & Females, $\mathbf{n} / \mathbf{N}(\%)$ & Repair & Replace & Postoperative LOS, days, median (range) & 30-d mortality, n/N $(\%)$ \\
\hline 2012 & 5 & $3 / 5(60)$ & 0 & 5 & $7(5-12)$ & $0 / 5$ \\
2013 & 9 & $4 / 9(44)$ & 1 & 8 & $42(25-42)$ & $1 / 9(11)$ \\
\hline 2014 & 1 & $1 / 1(100)$ & 0 & 1 & 32 & $0 / 1$ \\
2015 & 3 & $2 / 3(67)$ & 1 & 2 & $15(10.5-16.5)$ & $0 / 3$ \\
2016 & 13 & $8 / 13(62)$ & 7 & 6 & $42(21-42)$ & $0 / 13$ \\
2017 & 34 & $24 / 34(71)$ & 17 & 17 & $42(27-43)$ & $1 / 34(3)$ \\
2018 & 84 & $53 / 84(63)$ & 41 & 43 & $35(23-47)$ & $3 / 79(4)$ \\
Total & 149 & $95 / 149(64)$ & $67 / 149(45)$ & $82 / 149(55)$ & $37(22.5-43)$ & $5 / 144(3)$ \\
\hline
\end{tabular}

LOS, Length of stay. 


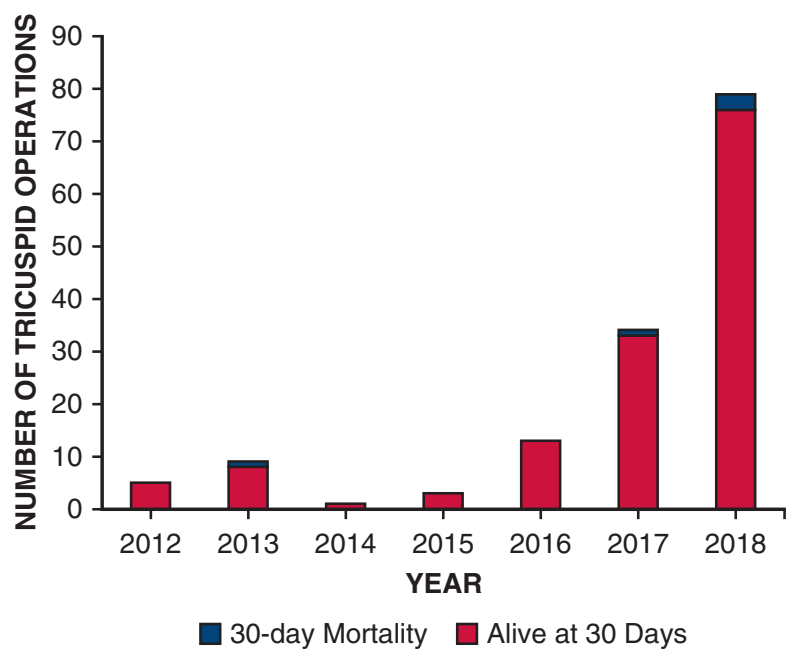

FIGURE 1. Incidence and outcomes of tricuspid valve operations for acute intravenous drug- associated endocarditis.

The median LOS ( 34 days vs 7 days; $P<.001$ ) and total hospital charges $(\$ 291,037$ vs $\$ 132,140 ; P<.001)$ were greater for patients with IVDA-related endocarditis (Figure 2).

In addition, patients undergoing endocarditis operations with IVDA $(\mathrm{n}=187)$ and without IVDA $(\mathrm{n}=71)$ were temporally compared. Patients with IVDA accounted for $31 \%$ of all endocarditis operations in 2012, which increased to $83 \%$ in 2018 . The median postoperative LOS was longer for patients with IVDA endocarditis compared with that for patients with non-IVDA endocarditis (34 days vs 11 days; $P<.001)$. The median overall charges were greater for IVDA endocarditis operations compared with nonIVDA endocarditis operations (\$291,037 vs $\$ 235,620$; $P=.048$ ), whereas median reimbursement was less for IVDA-related procedures $(\$ 42,063$ vs $\$ 70,393 ; P<.001)$. This institutional experience illustrates that providing care for patients with IVDA endocarditis represents a major unsustainable financial burden to the healthcare system. Patients requiring operations present acutely, often require noncardiac procedures related to their disease, and may require increased LOS for IV antibiotic therapy and addiction counseling to avoid acute recidivism and early mortality. ${ }^{11}$ The use of early inpatient drug rehabilitation and involvement of multidisciplinary addiction medicine teams in afflicted communities have shown early promise in combating recidivism; however, resources to achieve this important goal are dwarfed by the ever-growing need.

Given the ongoing societal problems with access, addiction, and recidivism, the secondary epidemic of IVDA endocarditis presents a substantial operational, ethical, and financial challenge for health systems and physicians providing essential care for these patients. On May 16, 2019, the attorneys general of Iowa, Kansas, Maryland, and West Virginia filed suit against Purdue Pharma, makers of OxyContin, in an attempt to secure reparations for victims of the opioid crisis. This action has since been followed by several other states and institutions, with nearly 2000 lawsuits filed in federal courts as of June 30, 2019.

Despite these efforts, which may or may not directly impact the care of these patients, the incidence and complexity of the management of IVDA endocarditis

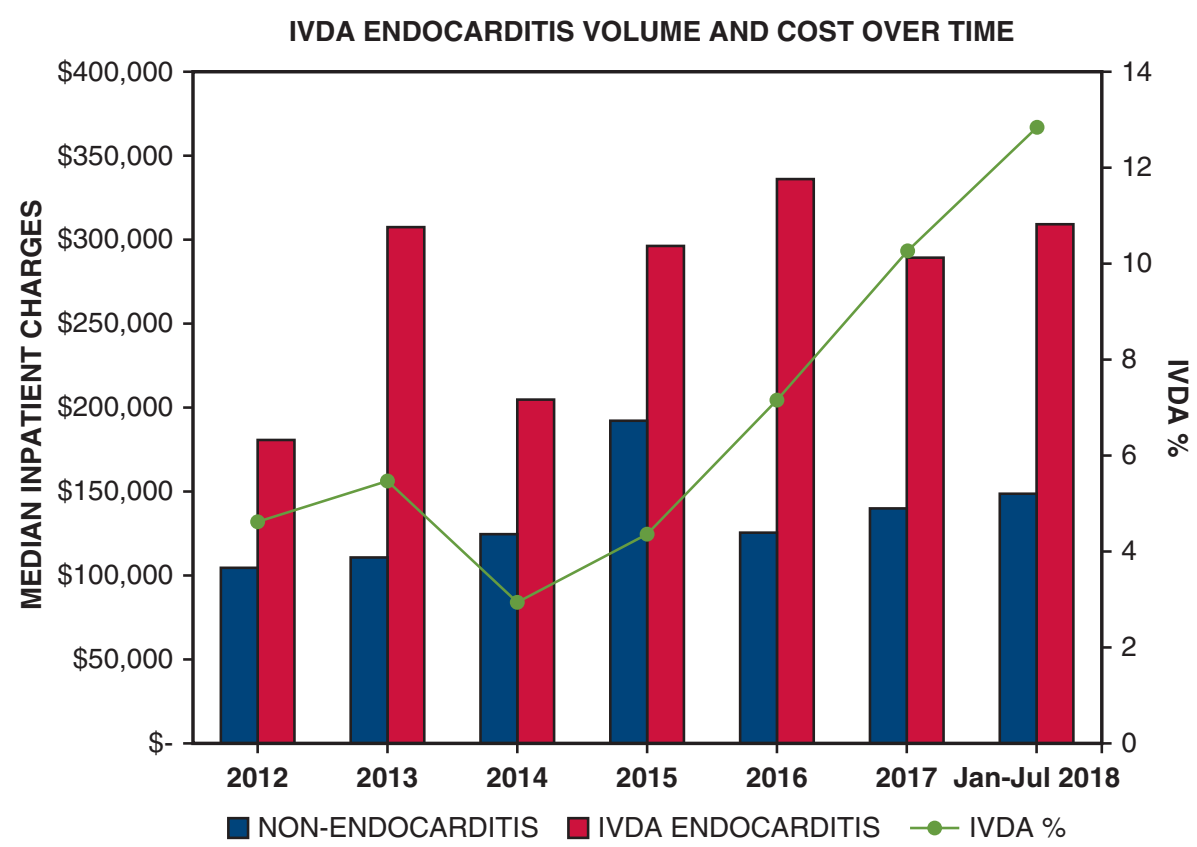

FIGURE 2. Intravenous drug-associated endocarditis, operative volume, and costs over time. Note that the 2018 data represent only the first 6 months. 
TABLE 2. Comparison of IVDA and non-IVDA endocarditis valve operations at West Virginia University

\begin{tabular}{|c|c|c|c|}
\hline Characteristic & IVDA endocarditis ( $N=187$ ) & Non-IVDA endocarditis $(N=613)$ & $P$ value \\
\hline Age, $y$, mean \pm SD & $33.8 \pm 9.0$ & $64.0 \pm 12.6$ & $<.001$ \\
\hline Female sex, $\mathrm{n}(\%)$ & $91(49)$ & $269(44)$ & .250 \\
\hline Elective surgery, n (\%) & $10(5)$ & 449 (73) & $<.001$ \\
\hline Aortic valve, n (\%) & $58(31)$ & $390(64)$ & $<.001$ \\
\hline Mitral valve, n (\%) & $60(32)$ & $254(41)$ & .022 \\
\hline Tricuspid valve, $\mathrm{n}(\%)$ & $103(55)$ & $54(9)$ & $<.001$ \\
\hline Pulmonic valve, n (\%) & $4(2)$ & $1(0.2)$ & .012 \\
\hline ICU LOS, h, median & 90.9 & 84.4 & .068 \\
\hline Postoperative LOS, d, median & 34 & 7 & $<.001$ \\
\hline Total actual charges, USD, median & 291,037 & 132,141 & $<.001$ \\
\hline Total reimbursement, USD, median & 42,063 & 40,841 & .331 \\
\hline Postoperative renal failure, $\mathrm{n}(\%)$ & $12(6)$ & $17(3)$ & .020 \\
\hline Prolonged ventilation (>24 h), n (\%) & $39(21)$ & $59(10)$ & $<.001$ \\
\hline Noncardiac reoperation, $\mathrm{n}(\%)$ & $16(9)$ & $17(3)$ & $<.001$ \\
\hline STS morbidity/mortality, n (\%) & $57(31)$ & $99(16)$ & $<.001$ \\
\hline Operative 30-d mortality, n (\%) & $6(3)$ & $20(3)$ & .971 \\
\hline
\end{tabular}

IVDA, Intravenous drug-associated; ICU, intensive care unit; $L O S$, length of stay; STS, Society of Thoracic Surgeons.

continues to rise. Nevertheless, a renewed collective focus of states, health systems, and payers is desperately needed to help stem the rising cost of care related to these desperate patients.

\section{HOPE FOR SOLUTIONS}

A recent randomized, noninferiority, multicenter trial published in the New England Journal of Medicine suggested a potentially important cost-saving strategy for the management of endocarditis. ${ }^{15}$ This Danish study randomized 400 adults in stable condition who had left-sided endocarditis caused by Streptococcus, Enterococcus faecalis, Staphylococcus aureus (methicillin-sensitive and methicillin-resistant), or coagulase-negative staphylococci and who were being treated with IV antibiotics to either a full course of IV antibiotic therapy or switching to oral antibiotic treatment after at least 10 days of IV antibiotics. Before randomization, 152 of the patients ( $38 \%$ ) had undergone valve surgery. In addition, patients with prosthetic valve endocarditis were included. This study concluded that in patients with left-sided endocarditis in stable condition, changing to oral antibiotic treatment after a median of 19 days of IV antibiotics was not inferior to continued IV antibiotic treatment in terms of a composite outcome or all-cause mortality, unplanned cardiac surgery, embolic event, and relapsed blood culture. ${ }^{15}$ In the same issue of the New England Journal of Medicine, investigators reported that oral antibiotic therapy was not inferior to IV antibiotic therapy when administered during the first 6 weeks for complex orthopedic infection as assessed by treatment failure at 1 year. ${ }^{16}$ Of course, both of these studies were performed in patients with non-IVDA endocarditis; nevertheless, the results provide encouraging evidence that in selected cases, oral antibiotic therapy may be effective for managing endocarditis that has been historically relegated to 6 weeks of IV antibiotic therapy by current guidelines (class of recommendation II, level of evidence C)..$^{17}$

The current management of acute IVDA endocarditis is clearly associated with unique challenges. Operations are often urgent or emergent due to associated acute secondary hemodynamic embarrassment. Operative complexity is often increased due to multivalve or intracardiac abscess involvement. Postoperative challenges include the need for additional noncardiac procedures, the use of indwelling catheters to deliver prolonged parenteral antibiotics to patients addicted to IV drugs, and the need for ample time for detoxification and addiction counseling to prevent recidivism. Despite the widely held perception that peripherally inserted central catheters are less morbid than traditional central lines, their complication profiles are not dissimilar and life-threatening problems can occur. ${ }^{18}$ The management and cost of the appropriate use of indwelling peripheral catheters in IV drug-abusing patients is not trivial. Following successful complex valve operations, patients with incompletely or untreated addiction discharged with these catheters for prolonged IV antibiotics may be often complicated by acute recidivism and early postoperative mortality. For this reason, many institutions have recently adopted a policy of inpatient management until the IV antibiotic course is complete. This prolonged hospitalization period provides time for detoxification, 
transition from postoperative narcotic pain management to partial opioid antagonists such as buprenorphine for addiction maintenance, and commencement of a crucial component of inpatient drug rehabilitation. ${ }^{11}$ This ethically and medically sound policy has an impact on healthcare costs that is unstainable, however. Moreover, once patients have recovered from a successful operation, the addiction and social issues that led to IV drug abuse may remain. These issues may be significant, because many patients refuse further treatment and sign out of the hospital against medical advice. It is not uncommon for patients to bargain to leave the hospital and even to request permission to take oral antibiotics and attempt to enroll in an inpatient drug rehabilitation program closer to home.

Early transition to oral antibiotic therapy in the postoperative management of endocarditis may provide several advantages. Should this be found to be safely applicable to postoperative IVDA endocarditis patients, these benefits may be further compounded. Advantages include a reduction in catheter-related complications, decreased LOS, an earlier return to normal function, and reduced cost. Should an increase in compliance be facilitated with simpler oral administration, this would facilitate earlier intensive addiction counseling and drug rehabilitation. Of course, such an option might not be appropriate for all patients with IVDA endocarditis, such as those in whom secondary noncardiac infection may persist (solid organs or joints), or for those with more virulent organisms, such as Serratia or HACEK infections (Haemophilus, Aggregatibacter, Cardiobacterium, Eikenella, Kingella).

Based on lessons from the Danish randomized trial, ${ }^{15}$ a similarly structured multicenter randomized trial is being planned with participating valve centers in West Virginia, Virginia, and Kentucky. Postoperative patients who have undergone valve repair or replacement for IVDA endocarditis may be randomized into two arms: conventional 6 weeks of postoperative IV antibiotics versus 3 weeks of IV antibiotics followed by 3 weeks of oral antibiotics. Both groups will receive aggressive drug rehabilitation, monitoring of treatment efficacy with serum antibiotic levels, as well as a compliance tracking tool for medication administration. Inclusion criteria will be patients receiving urgent/emergent valve operation who are blood culture-positive for Streptococcus, E faecalis, $S$ aureus, or coagulase-negative staphylococci, and in whom no residual signs of active imaging-confirmed infection exist at the time of postoperative randomization. Exclusion criteria would include concomitant infection requiring IV antibiotic therapy, inability to give informed consent, known poor compliance, and reduced absorption or inability to receive oral treatment due to a gastrointestinal disorder. The primary outcome measure would be all-cause mortality, recurrent blood culture-positive infection, and cardiac reoperation at 6 months. Secondary outcome measures would include 12-month all-cause mortality, readmission for recurrent infection, cardiac reoperation, and cost.

Prevention of recidivism is essential for the management of patients with IVDA-related endocarditis. ${ }^{14,19,20}$ The need for short-term reoperations for recurrent IVDA endocarditis strains the ethical, social, and financial foundations of health care. The creation of IVDA-related endocarditis heart teams involving surgeons, cardiologists, infectious disease specialists, drug addiction psychiatrists, social workers, medical ethicists, and palliative care specialists may cultivate pathways of evidence-based management for patients with IVDA-related endocarditis by facilitating early diagnosis, enhanced treatment, and reduced recidivism. ${ }^{11}$ Strategies should include aggressive antimicrobial and surgical therapy, followed by engaging regional systems of inpatient and outpatient drug rehabilitation and social worker home follow-up in an attempt to mitigate medical noncompliance and recidivism. In addition to preventive approaches, novel treatment strategies for patients with IVDA-related endocarditis, including the creation of IVDA-related endocarditis heart teams and early transition to oral antibiotics, if proven effective, may offer potential solutions to the opioid epidemic and IVDA endocarditis.

\section{Conflict of Interest Statement}

Dr Rankin is a consultant for AtriCure and BioStable. Dr Ailawadi is a consultant for Edwards and Abbott. Dr Badhwar is a consultant for Abbott (uncompensated). All other authors have nothing to disclose with regard to commercial support.

\section{References}

1. Centers for Disease Control and Prevention. Drug overdose deaths. Available at: https://www.cdc.gov/drugoverdose/data/statedeaths.html. Accessed June 10, 2019.

2. Scholl L, Seth P, Kariisa M, Wilson N, Baldwin G. Drug and opioid-involved overdose deaths - United States, 2013-2017. MMWR Morb Mortal Wkly Rep. 2019;67:1419-27.

3. Centers for Disease Control and Prevention. Wide-ranging Online Data for Epidemiologic Research (CDC WONDER): Multiple cause of death 1999-2017. Available at: http://wonder.cdc.gov. Accessed June 10, 2019.

4. Wurcel AG, Anderson JE, Chui KK, Skinner S, Knox TA, Snydman DR, et al. Increasing infectious endocarditis admissions among young people who inject drugs. Open Forum Infect Dis. 2016;3:ofw157.

5. Kolodny A, Courtwright DT, Hwang CS, Kreiner P, Eadie JL, Clark TW, et al. The prescription opioid and heroin crisis: a public health approach to an epidemic of addiction. Annu Rev Public Health. 2015;36:559-74.

6. Marks RM, Sachar EJ. Undertreatment of medical inpatients with narcotic analgesics. Ann Intern Med. 1973;78:173-81.

7. Porter J, Jick H. Addiction rare in patients treated with narcotics. $N$ Engl J Med. 1980;302:123.

8. American Academy of Pain Medicine; American Pain Society. The use of opioids for the treatment of chronic pain. A consensus statement from the American Academy of Pain Medicine and the American Pain Society. Clin J Pain. 1997;13:6-8.

9. Tompkins DA, Hobelmann JG, Compton P. Providing chronic pain management in the "fifth vital sign" era: historical and treatment perspectives 
on a modern-day medical dilemma. Drug Alcohol Depend. 2017;173(Suppl 1): S11-21.

10. Ferraris VA, Sekela ME. Missing the forest for the trees: the world around us and surgical treatment of endocarditis. J Thorac Cardiovasc Surg. 2016;152:677-80.

11. Badhwar V, Wei LM, Rankin JS. Seeing the entire forest in endocarditis. J Thorac Cardiovasc Surg. 2016;152:681-2.

12. American Academy of Pain Medicine; American Pain Society; American Society of Addiction Medicine. Public policy statement on the rights and responsibilities of health care professionals in the use of opioids for the treatment of pain: a consensus document from the American Academy of Pain Medicine, the American Pain Society, and the American Society of Addiction Medicine. Pain Med. 2004;5:301-2.

13. Muhiri PK, Gfroerer JC, Davies MC. Associations of nonmedical pain reliever use and initiation of heroin use in the United States. CBHSQ Data Review, August 2013. Center for Behavioral Health Statistics and Quality, The Substance Abuse and Mental Health Services Administration (SAMHSA). Available at: https://www.samhsa.gov/data/. Accessed June 10, 2019.

14. Nguemeni Tiako MJ, Mori M, Bin Mahmood SU, Shioda K, Mangi A, Yun J, et al. Recidivism is the leading cause of death among intravenous drug users who underwent cardiac surgery for infective endocarditis. Semin Thorac Cardiovasc Surg. 2019;31:40-5.

15. Iversen K, Ihlemann N, Gill SU, Madsen T, Elming H, Jensen KT, et al. Partial oral versus intravenous antibiotic treatment of endocarditis. $N$ Engl J Med. 2019;380:415-24.

16. Li HK, Rombach I, Zambellas R, Walker AS, McNally MA, Atkins BL, et al. Oral versus intravenous antibiotics for bone and joint infection. $N$ Engl J Med. 2019;380:425-36.

17. Baddour LM, Wilson WR, Bayer AS, Fowler VG Jr, Tleyjeh IM, Rybak MJ, et al. Infective endocarditis in adults: diagnosis, antimicrobial therapy, and management of complications: a scientific statement for healthcare professionals from the American Heart Association. Circulation. 2015;132:1435-86.

18. Kornbau C, Lee KC, Hughes GD, Firstenberg MS. Central line complications. Int J Crit Illn Inj Sci. 2015;5:170-8.

19. Hull SC, Jadbabaie F. When is enough enough? The dilemma of valve replacement in a recidivist intravenous drug user. Ann Thorac Surg. 2014;97:1486-7.

20. Kim JB, Ejiofor JI, Yammine M, Ando M, Camuso JM, Youngster I, et al. Surgical outcomes of infective endocarditis among intravenous drug users. $J$ Thorac Cardiovasc Surg. 2016;152:832-41.e1. 